\title{
CARLESON MEASURES AND REPRODUCING KERNEL THESIS IN DIRICHLET-TYPE SPACES
}

\author{
G. R. CHACÓN, E. FRICAIN, AND M. SHABANKHAH
}

\begin{abstract}
In the paper, a generalization of a Richter and Sundberg representation theorem is employed to obtain a new characterization of Carleson measures for the Dirichlet-type space $\mathcal{D}(\mu)$ when $\mu$ is a finite sum of point masses. A reproducing kernel thesis result is also established in this case.
\end{abstract}

\section{§1. INTRODUCTION}

Dirichlet-type spaces (also called local Dirichlet spaces) have been introduced by S. Richter [21] when he investigated analytic two-isometries. This class of operators appeared for the first time in [1] in connection with the compression of a first-order differential operator to the Hardy space $H^{2}$ on the unit disk. The study of two-isometries and related operators is also of interest for its links with the theory of dilations and invariant subspaces of the shift operator on the classical Dirichlet space $\mathcal{D}[19$. It is an immediate consequence of the norm definitions that $M_{z}$, the operator of multiplication by the independent variable $z$, is an isometry on $H^{2}$ but not on $\mathcal{D}$ (see $\S 2$ for precise definitions). In fact, one can verify that $M_{z}$ is an analytic two-isometry on $\mathcal{D}$. It is a remarkable result of S. Richter [21] that every analytic two-isometry satisfying $\operatorname{dim} \operatorname{Ker}\left(T^{*}\right)=1$ is unitarily equivalent to $M_{z}$ on some Dirichlet-type space $\mathcal{D}(\mu)$. These spaces have been studied ever since by several authors, see for example $[2,7,4,9,10,20,24,25,26,28,29$.

In particular, in [7, the first author introduced a notion of capacity adapted to Dirichlet-type spaces, and gave a characterization of Carleson measures for $\mathcal{D}(\mu)$ in terms of this capacity. Carleson measures for the Hardy space have proved to be objects of fundamental importance in the development of modern function theory. In particular, they have appeared in areas ranging from the celebrated Corona problem and its solution by Carleson [6], to the development of bounded mean oscillation (BMO) functions by C. Fefferman and E. Stein [12, P. Jones [15] and many others. The characterization obtained in [7] is similar to those given by D. Stegenga in [30] for the classical Dirichlet space. In the present paper, we will provide a new characterization of the Carleson measures for the space $\mathcal{D}(\mu)$ when $\mu$ is a finite sum of point masses. As we will see, in this case, Carleson measures for $\mathcal{D}(\mu)$ are determined, in a very specific way, those in terms of of $H^{2}$. The key idea is a generalization of the Richter-Sundberg representation theorem.

The other natural question we address is the reproducing kernel thesis for the embedding $\mathcal{D}(\mu) \hookrightarrow L^{2}(\nu)$, where $\nu$ is a positive Borel measure on the unit disk. Recall

2010 Mathematics Subject Classification. Primary 46E20, 46E22.

Key words and phrases. Dirichlet-type spaces, Carleson measures, reproducing kernel thesis.

The first author was partially supported by Pontifica Universidad Javeriana, project 4884. The second author was partially supported by the ANR FRAB. The third author was supported by ANR DYNOP and FQRNT. 
that an operator on a reproducing kernel Hilbert space is said to satisfy the Reproducing Kernel Thesis (RKT) if its boundedness is determined by its behavior on the reproducing kernels. In general, there is no reason why this should be true but it turns out, as was proved by L. Carleson, that this is indeed the case for the identity map $I: H^{2} \rightarrow L^{2}(\nu)$. More explicitly, $I$ is bounded (compact, respectively) on $H^{2}$ if and only if it acts as a bounded (compact, respectively) operator on the set $\left\{k_{z}, z \in \mathbb{D}\right\}$. Though there were many results of this type since Carleson's result, philosophically the idea to study (RKT) for classes of operators in general reproducing kernel Hilbert spaces comes from [14] (see also [18]). We will show that the identity map $I: \mathcal{D}(\mu) \rightarrow L^{2}(\nu)$ is another example of operators satisfying the (RKT), in the case where $\mu$ is a finite sum of point masses. We mention that there is another natural generalization of the classical Dirichlet space $\mathcal{D}$, the so-called weighted Dirichlet spaces, where the (RKT) for the embedding in $L^{2}(\nu)$ space is not valid (see Remark 6.3 for further details).

The plan of the paper is the following. The next section contains preliminary material concerning Dirichlet-type spaces and Carleson measures. $\S 3$ contains a representation theorem for functions in $\mathcal{D}(\mu)$ spaces corresponding to the case where $\mu$ is a finite sum of point masses. In $\S 4$, we give a new characterization of Carleson measures in Dirichlet-type spaces induced by finitely atomic measures. In $\S \S 5$ and 6 , a reproducing kernel thesis for the embedding $\mathcal{D}(\mu) \hookrightarrow L^{2}(\nu)$ is established. Finally, in $\S 7$, compact Carleson measures for Dirichlet-type spaces induced by finitely atomic measures are characterized in terms of the normalized reproducing kernels of the space.

\section{§2. Preliminaries}

Recall that the Hardy space $H^{2}$ of the unit disk $\mathbb{D}=\{z \in \mathbb{C}:|z|<1\}$ is the Hilbert space of functions $f(z)=\sum_{n \geq 0} a_{n} z^{n}$ analytic on $\mathbb{D}$ and such that

$$
\|f\|_{2}^{2}:=\sum_{n \geq 0}\left|a_{n}\right|^{2}<+\infty
$$

If $f$ is an analytic function on $\mathbb{D}$, then we denote by $\mathcal{D}(f)$ its Dirichlet integral given by

$$
\mathcal{D}(f):=\int_{\mathbb{D}}\left|f^{\prime}(z)\right|^{2} d A(z),
$$

where $d A$ is the normalized area measure. Then the classical Dirichlet space $\mathcal{D}$ consists of analytic functions whose Dirichlet integral is finite. It is easily verified that $\mathcal{D}$ is a subspace of $H^{2}$. In particular, $\mathcal{D}$ turns into a Hilbert space under the following natural norm:

$$
\|f\|_{\mathcal{D}}^{2}:=\|f\|_{2}^{2}+\mathcal{D}(f)
$$

Now let $\mu$ be a positive finite Borel measure on the unit circle $\mathbb{T}$, and let $P_{\mu}$ be its harmonic extension to $\mathbb{D}$, i.e.,

$$
P_{\mu}(z)=\int_{\mathbb{T}} \frac{1-|z|^{2}}{|\zeta-z|^{2}} d \mu(\zeta), \quad z \in \mathbb{D} .
$$

The corresponding $\mathcal{D}(\mu)$-space is defined to be the set of holomorphic functions $f$ for which

$$
\mathcal{D}_{\mu}(f):=\int_{\mathbb{D}}\left|f^{\prime}(z)\right|^{2} P_{\mu}(z) d A(z)<\infty .
$$

In particular, if $\mu$ is taken to be the normalized Lebesgue measure on $\mathbb{T}$, then $P_{\mu}(z)=1$, $z \in \mathbb{D}$, and therefore $\mathcal{D}(\mu)=\mathcal{D}$, the classical Dirichlet space. The quantity $\mathcal{D}_{\mu}(f)$ is called the Dirichlet integral of $f$ with respect to $\mu$. If $\mu=\delta_{\lambda}$, i.e., the unit point mass 
at $\lambda \in \mathbb{T}$, one writes $\mathcal{D}_{\lambda}(f)$ instead of $\mathcal{D}_{\mu}(f)$, and calls it the local Dirichlet integral of $f$ at $\lambda$. By Fubini's Theorem, we have

$$
\mathcal{D}_{\mu}(f)=\int_{\mathbb{T}} \mathcal{D}_{\lambda}(f) d \mu(\lambda)
$$

In 22 S. Richter and C. Sundberg proved that $f \in \mathcal{D}\left(\delta_{\lambda}\right)$ if and only if $f=c+(z-\lambda) g$, where $c$ is a constant and $g \in H^{2}$. In this case, $c$ is the non-tangential (even oricyclic) limit of $f$ at $\lambda$, denoted by $f(\lambda)$, and $\|g\|_{2}^{2}=\mathcal{D}_{\lambda}(f)$. In other words,

$$
\mathcal{D}_{\lambda}(f)=\left\|\frac{f-f(\lambda)}{z-\lambda}\right\|_{2}^{2} .
$$

A different proof of these facts can be found in 24. Since $\mathcal{D}\left(\delta_{\lambda}\right) \subset H^{2}$, from (2.1) it follows that $\mathcal{D}(\mu) \subset H^{2}$. The norm with respect to which $\mathcal{D}(\mu)$ is a Hilbert space, is given by

$$
\|f\|_{\mu}^{2}:=\|f\|_{2}^{2}+\mathcal{D}_{\mu}(f) .
$$

Moreover, polynomials form a dense subset of $\mathcal{D}(\mu)$ 21].

The Hardy space $H^{2}$ is an example of a reproducing kernel Hilbert space, i.e., the point evaluation $f \mapsto f(z)$ is a bounded functional on $H^{2}$, for every $z \in \mathbb{D}$. In particular,

$$
f(z)=\left\langle f, k_{z}\right\rangle_{2},
$$

for some $k_{z} \in H^{2}$. One calls $k_{z}$ the reproducing kernel at $z$. By (2.3), the point evaluation $f \mapsto f(z)$ is also a bounded functional on $\mathcal{D}(\mu)$, for every $z \in \mathbb{D}$. Now, if $\lambda \in \mathbb{T}$, using the uniform boundedness principle, we easily see that the functional $f \mapsto f(\lambda)$ is bounded on $\mathcal{D}\left(\delta_{\lambda}\right)$ (whereas it is never bounded on $H^{2}$ ).

An important notion in the theory of complex function spaces is that of Carleson measures. Given a Banach space $X$ of holomorphic functions in $\mathbb{D}$, one says that $\nu$, a positive Borel measure on $\mathbb{D}$, is a Carleson measure for $X$ if the identity map $I: X \rightarrow L^{2}(\nu)$ is a bounded operator, i.e., there is a constant $C>0$ such that

$$
\int_{\mathbb{D}}|f|^{2} d \nu \leq C\|f\|_{X}^{2}
$$

for every function $f$ in $X$. If the embedding happens to be compact, then $\nu$ is called a compact (or vanishing) Carleson measure.

In the setting of the Hardy space $H^{2}$, a geometric characterization of the corresponding measures was obtained by L. Carleson. He proved that $\nu$ is a Carleson measure for $H^{2}$ if and only if

$$
\nu(S(\zeta, h))=O(h), \quad h \rightarrow 0^{+},
$$

where, for $\zeta \in \mathbb{T}$ and $0<h<1$, the set $S(\zeta, h)$ is given by

$$
S(\zeta, h):=\left\{z \in \mathbb{D}: 1-h<|z|<1 \text { and }\left|\frac{z}{|z|}-\zeta\right|<\frac{h}{2}\right\} .
$$

See for example [13] for a proof of this classical result. As one might expect, the characterization of compact Carleson measures for $H^{2}$ is obtained by replacing " $O$ " in the above condition by " $O$ ".

\section{§3. A representation Theorem}

The following result extends the Richter-Sundberg representation theorem [22] to the case of finitely atomic measures. A part of this result already appeared (at least implicitly) in [25]. 
Theorem 3.1. Let $\mu=\sum_{j=1}^{n} \alpha_{j} \delta_{\lambda_{j}}$ be a finitely atomic measure, $\alpha_{j}>0, \lambda_{j} \in \mathbb{T}$, $1 \leq j \leq n$. Then $f \in \mathcal{D}(\mu)$ if and only if there exists $g \in H^{2}$ and a polynomial $p$, $\operatorname{deg} p \leq n-1$, such that

$$
f=p+\prod_{j=1}^{n}\left(z-\lambda_{j}\right) g .
$$

Moreover, $g$ and $p$ are unique, and we have

$$
\|g\|_{2} \leq C\|f\|_{\mu}
$$

for some positive constant $C=C(n, \mu)$.

Proof. If $f$ is given by (3.1), then $f \in \mathcal{D}(\mu)$. Indeed, we have

$$
\prod_{j=1}^{n}\left(z-\lambda_{j}\right) g \in \bigcap_{j=1}^{n} \mathcal{D}\left(\delta_{\lambda_{j}}\right)=\mathcal{D}(\mu) .
$$

Now let $f \in \mathcal{D}(\mu)$. We will show that $f$ has a representation of the form (3.1). The case of $n=1$ being already established in [22], we consider first the case where $n=2$. Let $f \in \mathcal{D}(\mu)$. Suppose that $f\left(\lambda_{1}\right)=f\left(\lambda_{2}\right)=0$. Since $f \in \mathcal{D}\left(\delta_{\lambda_{1}}\right) \cap \mathcal{D}\left(\delta_{\lambda_{2}}\right)$, we have

$$
f=\left(z-\lambda_{1}\right) g_{1}=\left(z-\lambda_{2}\right) g_{2},
$$

for some $g_{1}, g_{2} \in H^{2}$. A simple calculation shows that $g_{1} /\left(z-\lambda_{2}\right) \in H^{2}$. In particular, $f$ can be written as

$$
f=\left(z-\lambda_{1}\right)\left(z-\lambda_{2}\right) g
$$

where $g \in H^{2}$. Using induction, we see that if $f \in \mathcal{D}(\mu)$ with $f\left(\lambda_{j}\right)=0,1 \leq j \leq n$, then

$$
f=\left(z-\lambda_{1}\right) \cdots\left(z-\lambda_{n}\right) g,
$$

for some $g \in H^{2}$. For the general case, we choose a polynomial $p$ of degree less than or equal to $n-1$ and such that

$$
p\left(\lambda_{j}\right)=f\left(\lambda_{j}\right), \quad 1 \leq j \leq n .
$$

Upon applying the preceding argument to $f-p$, we obtain (3.1).

For the uniqueness in the decomposition (3.1), we simply note that the polynomial $p$ is necessarily given by

$$
p(z)=\sum_{j=1}^{n} \prod_{\substack{1 \leq k \leq n, k \neq j}} \frac{z-\lambda_{k}}{\lambda_{j}-\lambda_{k}} f\left(\lambda_{j}\right) .
$$

It remains to prove the norm inequality (3.2). Since the evaluation functionals $f \mapsto$ $f\left(\lambda_{j}\right)$ are bounded, $1 \leq j \leq n$, we see that

$$
\|p\|_{\mu}^{2}=\|p\|_{2}^{2}+\int_{\mathbb{D}}\left|p^{\prime}(z)\right|^{2} P_{\mu}(z) d A(z) \leq C\|f\|_{\mu}^{2},
$$

where $C$ is a constant depending on $n$ and $\mu$. In particular, by the triangle inequality,

$$
\left\|\prod_{j=1}^{n}\left(z-\lambda_{j}\right) g\right\|_{\mu} \leq C\|f\|_{\mu} .
$$

To complete the proof of (3.2), we need to show that

$$
\|g\|_{2} \leq C\left\|\prod_{j=1}^{n}\left(z-\lambda_{j}\right) g\right\|_{\mu}
$$


This is obvious if $n=1$. In fact, given $\mu=\alpha_{1} \delta_{\lambda_{1}}$, by (2.1) and (2.3) we have

$$
\begin{aligned}
\left\|\left(z-\lambda_{1}\right) g\right\|_{\mu}^{2}=\left\|\left(z-\lambda_{1}\right) g\right\|_{2}^{2}+\mathcal{D}_{\mu}\left(\left(z-\lambda_{1}\right) g\right) & =\left\|\left(z-\lambda_{1}\right) g\right\|_{2}^{2}+\alpha_{1} \mathcal{D}_{\lambda_{1}}\left(\left(z-\lambda_{1}\right) g\right) \\
& =\left\|\left(z-\lambda_{1}\right) g\right\|_{2}^{2}+\alpha_{1}\|g\|_{2}^{2} \geq \alpha_{1}\|g\|_{2}^{2} .
\end{aligned}
$$

For $n=2$, we have

$$
\left|\lambda_{1}-\lambda_{2}\right|^{2}\|g\|_{2}^{2} \leq 2\left(\left\|\left(z-\lambda_{1}\right) g\right\|_{2}^{2}+\left\|\left(z-\lambda_{2}\right) g\right\|_{2}^{2}\right) \leq 2\left\|\prod_{j=1}^{2}\left(z-\lambda_{j}\right) g\right\|_{\mu}^{2} .
$$

The general case follows by induction.

\section{$\S 4$. Carleson measures}

Theorem 3.1 enables us to give a complete description of Carleson measures in Dirichlet spaces induced by finitely atomic measures.

Theorem 4.1. Let $\mu=\sum_{j=1}^{n} \alpha_{j} \delta_{\lambda_{j}}$ be a finitely atomic measure, $\alpha_{j}>0, \lambda_{j} \in \mathbb{T}$, $1 \leq j \leq n$. A finite positive Borel measure $\nu$ on $\mathbb{D}$ is a Carleson measure for $\mathcal{D}(\mu)$ if and only if $\prod_{j=1}^{n}\left|z-\lambda_{j}\right|^{2} d \nu(z)$ is a Carleson measure for $H^{2}$.

Proof. Assume that $\nu$ is a Carleson measure for $\mathcal{D}(\mu)$. Since for every $g \in H^{2}$ we have $f=\prod_{j=1}^{n}\left(z-\lambda_{j}\right) g \in \mathcal{D}(\mu)$, it follows that

$$
\int_{\mathbb{D}} \prod_{j=1}^{n}\left|z-\lambda_{j}\right|^{2}|g|^{2} d \nu(z) \leq C\|f\|_{\mu}^{2} \leq C^{\prime}\|g\|_{2}^{2}
$$

where the last inequality is an easy consequence of the definition of the norm in $\mathcal{D}(\mu)$. Consequently, $\prod_{j=1}^{n}\left|z-\lambda_{j}\right|^{2} d \nu(z)$ is a Carleson measure for $H^{2}$.

Conversely, if $\prod_{j=1}^{n}\left|z-\lambda_{j}\right|^{2} d \nu(z)$ is a Carleson measure for $H^{2}$, we will show that $\nu$ is a Carleson measure for $\mathcal{D}(\mu)$. Let $f \in \mathcal{D}(\mu)$. By Theorem 3.1 .

$$
f=p+\prod_{j=1}^{n}\left(z-\lambda_{j}\right) g
$$

where $g \in H^{2}$ and $p$ is given by (3.3). Since the evaluation functionals $f \mapsto f\left(\lambda_{j}\right)$, $1 \leq j \leq n$, are bounded on $\mathcal{D}(\mu)$, we see that

$$
\int_{\mathbb{D}}|f|^{2} d \nu \leq C\left(\int_{\mathbb{D}}|p|^{2} d \nu+\int_{\mathbb{D}} \prod_{j=1}^{n}\left|z-\lambda_{j}\right|^{2}|g|^{2} d \nu\right) \leq C\left(\|f\|_{\mu}^{2}+\|g\|_{2}^{2}\right) .
$$

An appeal to (3.2) yields the desired conclusion.

Example 4.2. It is clear that every Carleson measure for $H^{2}$ is also a Carleson measure for $\mathcal{D}(\mu)$. The converse, however, is not true. Let $\alpha \in(0,1)$, and let

$$
d \nu(z)=(1-|z|)^{-\alpha} d m(z),
$$

where $d m$ is the Lebesgue measure on $[0,1)$. Since $d m$ is a Carleson measure for $H^{2}$, the preceding result implies that $d \nu$ is indeed a Carleson measure for $\mathcal{D}\left(\delta_{1}\right)$. We claim that $d \nu$ is not a Carleson measure for $H^{2}$. For $\zeta \in \mathbb{T}$ and $0<h<1$, consider $S(\zeta, h)$ as given by (2.5). If $S(\zeta, h) \cap[0,1) \neq \varnothing$, then

$$
\nu(S(\zeta, h))=\int_{1-h}^{1} \frac{d r}{(1-r)^{\alpha}}=\frac{h^{1-\alpha}}{1-\alpha},
$$


which proves the claim because

$$
\lim _{h \rightarrow 0^{+}} \frac{\nu(S(\zeta, h))}{h}=\infty
$$

In fact, given $0<\beta<1$, if we choose $\alpha$ such that $1-\beta<\alpha<1$, then the same example gives a Carleson measure $\nu$ for $\mathcal{D}\left(\delta_{1}\right)$ that satisfies $\nu(S(\zeta, h)) / h^{\beta} \rightarrow+\infty$, as $h \rightarrow 0^{+}$. This means that we cannot translate directly our characterization of Carleson measures for $\mathcal{D}(\mu)$ in terms of the asymptotic of $\nu(S(\zeta, h))$.

As the following result shows, compact Carleson measures for $\mathcal{D}(\mu)$ are characterized in a similar manner.

Theorem 4.3. Let $\mu=\sum_{j=1}^{n} \alpha_{j} \delta_{\lambda_{j}}$ be a finitely atomic measure. A finite positive Borel measure $\nu$ is a compact Carleson measure for $\mathcal{D}(\mu)$ if and only if $\prod_{j=1}^{n}\left|z-\lambda_{j}\right|^{2} d \nu(z)$ is a compact Carleson measure for $\mathrm{H}^{2}$.

Proof. Suppose that $\nu$ is a compact Carleson measure for $\mathcal{D}(\mu)$. Let $\left(g_{k}\right)_{k \geq 1} \subset H^{2}$ be such that $\left(g_{k}\right)_{k}$ converges weakly to zero, as $k \rightarrow \infty$. Put $f_{k}=\prod_{j=1}^{n}\left(z-\lambda_{j}\right) g_{k}, k \geq 1$. Since the operator $T: H^{2} \rightarrow \mathcal{D}(\mu)$ of multiplication by $\prod_{j=1}^{n}\left(z-\lambda_{j}\right)$ is bounded, it follows that $f_{k} \rightarrow 0$ weakly in $\mathcal{D}(\mu)$. So, the assumption that $I: \mathcal{D}(\mu) \rightarrow L^{2}(\nu)$ is compact implies that

$$
\int_{\mathbb{D}}\left|f_{k}\right|^{2} d \nu(z)=\int_{\mathbb{D}}\left|g_{k}\right|^{2} \prod_{j=1}^{n}\left|z-\lambda_{j}\right|^{2} d \nu(z) \rightarrow 0, \quad k \rightarrow \infty .
$$

Therefore, $\prod_{j=1}^{n}\left|z-\lambda_{j}\right|^{2} d \nu(z)$ is a compact Carleson measure for $H^{2}$.

For the converse, assume that $\left(f_{k}\right)_{k}$ is a sequence in $\mathcal{D}(\mu)$ that converges weakly to zero. We will show that $\left\|f_{k}\right\|_{L^{2}(\nu)} \rightarrow 0$, as $k \rightarrow \infty$. Indeed, by Theorem (3.1), $f_{k}=p_{k}+\prod_{j=1}^{n}\left(z-\lambda_{j}\right) g_{k}$, where $g_{k} \in H^{2}$ and

$$
p_{k}(z)=\sum_{j=1}^{n} \prod_{m \neq j} \frac{z-\lambda_{m}}{\lambda_{j}-\lambda_{m}} f_{k}\left(\lambda_{j}\right), \quad k \geq 1 .
$$

By (3.2), we see that $\left(g_{k}\right)_{k}$ is a bounded sequence in $H^{2}$. Moreover, since the evaluation functionals $f \mapsto f\left(\lambda_{j}\right)$ are bounded on $\mathcal{D}(\mu)$, for $1 \leq j \leq n$, and the sequence $f_{k}$ converges to zero uniformly on compact subsets of $\mathbb{D}$, we see that $g_{k} \rightarrow 0$ uniformly on compacta. In other words, $\left(g_{k}\right)_{k}$ is a sequence converging weakly to zero in $H^{2}$. Hence,

$$
\begin{aligned}
\int_{\mathbb{D}}\left|f_{k}\right|^{2} d \nu & \leq C\left(\int_{\mathbb{D}}\left|p_{k}(z)\right|^{2} d \nu(z)+\int_{\mathbb{D}}\left|g_{k}\right|^{2} \prod_{j=1}^{n}\left|z-\lambda_{j}\right|^{2} d \nu(z)\right) \\
& \leq C\left(\sum_{j=1}^{n}\left|f_{k}\left(\lambda_{j}\right)\right|^{2}+\int_{\mathbb{D}}\left|g_{k}\right|^{2}, \prod_{j=1}^{n}\left|z-\lambda_{j}\right|^{2} d \nu(z)\right) \rightarrow 0
\end{aligned}
$$

as $k \rightarrow \infty$. The proof is now complete.

Remark 4.4. In [10], R. Chartrand introduces a notion of a Carleson type measure in a different way than the one used here and in [7. In fact, in [7, exhibiting two examples, it was proved that the two definitions are really different. 


\section{$\S 5$. Reproducing Kernel thesis FOR A ONE POINT MASS}

Dirichlet-type spaces $\mathcal{D}(\mu)$ are reproducing kernel Hilbert spaces. Here, we will prove a reproducing kernel thesis result in the case when $\mu$ is a finite sum of point masses (Theorem 5.1 and Theorem [6.1). One of the difficulties here is to obtain an explicit expression for the reproducing kernels. As far as we know, except for the case of $\mathcal{D}\left(\delta_{\lambda}\right)$, such an explicit expression is not known.

Improving an earlier result of Richter and Sundberg 22, Sarason 24] showed that if $\mu=\delta_{\lambda}$, then $\mathcal{D}(\mu)$ can be identified with a de Branges-Rovnyak space. More precisely, Sarason proved that $\mathcal{D}\left(\delta_{\lambda}\right)=\mathcal{H}\left(b_{\lambda}\right)$, with equality of norms, where

$$
b_{\lambda}(z)=\frac{\left(1-a_{0}\right) \bar{\lambda} z}{1-a_{0} \bar{\lambda} z}, \quad z \in \mathbb{D},
$$

where $a_{0}$ is the smallest root of $\left(a_{0}-1\right)^{2}=a_{0}$. Recall here that, given $b$ in the closed unit ball of $H^{\infty}$, the de Branges-Rovnyak space $\mathcal{H}(b)$ is defined as the range of the operator $\left(I-T_{b} T_{b}^{*}\right)^{1 / 2}$, equipped with the range norm, where $T_{b}$ denotes the Toeplitz operator on $H^{2}$ (which, in this case, corresponds to the operator of multiplication by $b$ on $H^{2}$ ). Then we know [23] that $\mathcal{H}(b)$ is a reproducing kernel Hilbert space, whose reproducing kernel functions are given by

$$
k_{w}^{b}(z)=\frac{1-\overline{b(w)} b(z)}{1-\bar{w} z}, \quad w, z \in \mathbb{D} .
$$

Now if $\mu=\alpha \delta_{\lambda}$ with $\alpha>0$, then one still has $\mathcal{D}(\mu)=\mathcal{H}\left(b_{\lambda}\right)$, except that $a_{0}$ in (5.1) must be replaced by the smallest root of the equation $\left(a_{0}-1\right)^{2}=\alpha a_{0}$. This was in fact proved in [11] where it was also shown that this is the only case where $\mathcal{D}(\mu)$ arises as a de Branges-Rovnyak space (see also [8]).

Using this identification, we first prove the following reproducing kernel thesis in the special case of a point mass.

Theorem 5.1. Let $\nu$ be a finite positive Borel measure on $\mathbb{D}$, and let $\mu=\alpha \delta_{\lambda}$, where $\lambda \in \mathbb{T}$ and $\alpha>0$. Then $\nu$ is a Carleson measure for $\mathcal{D}(\mu)$ if and only if there exists a constant $C>0$ such that

$$
\int_{\mathbb{D}}\left|k_{w}^{\mu}\right|^{2} d \nu \leq C\left\|k_{w}^{\mu}\right\|_{\mu}^{2}
$$

for every $w \in \mathbb{D}$, where $k_{w}^{\mu}$ is the reproducing kernel for $\mathcal{D}(\mu)$ at $w$.

Proof. Suppose that (5.2) holds true. By the paragraph preceding the theorem, we have

$$
\int_{\mathbb{D}}\left|\frac{1-\overline{b_{\lambda}(w)} b_{\lambda}(z)}{1-\bar{w} z}\right|^{2} d \nu(z) \leq C \frac{1-\left|b_{\lambda}(w)\right|^{2}}{1-|w|^{2}}, \quad w \in \mathbb{D}
$$

where $b_{\lambda}$ is given by (5.1) and $a_{0} \in(0,1)$ satisfies $\left(a_{0}-1\right)^{2}=\alpha a_{0}$. We will show that (5.3) implies

$$
\int_{\mathbb{D}} \frac{1}{|1-\bar{w} z|^{2}}|z-\lambda|^{2} d \nu(z) \leq C_{1} \frac{1}{1-|w|^{2}}, \quad w \in \mathbb{D},
$$

for some positive constant $C_{1}$. Inequality (5.4), in conjunction with Carleson's reproducing kernel thesis for $H^{2}$, implies that $|z-\lambda|^{2} d \nu(z)$ is a Carleson measure for $H^{2}$. The desired conclusion then follows from Theorem 4.1 .

To prove (5.4), it suffices to show that

$$
\frac{1-|w|^{2}}{|1-\bar{w} z|^{2}}|z-\lambda|^{2} \leq C_{2}\left|\frac{1-\overline{b_{\lambda}(w)} b_{\lambda}(z)}{1-\bar{w} z}\right|^{2} \frac{1-|w|^{2}}{1-\left|b_{\lambda}(w)\right|^{2}}, \quad z, w \in \mathbb{D},
$$


or equivalently

$$
\inf _{z, w \in \mathbb{D}} \frac{\left|1-\overline{b_{\lambda}(w)} b_{\lambda}(z)\right|^{2}}{\left(1-\left|b_{\lambda}(w)\right|^{2}\right)|z-\lambda|^{2}}>0
$$

Using the inequality

$$
\frac{\left(1-|\gamma|^{2}\right)\left(1-\left.\beta\right|^{2}\right)}{|1-\bar{\gamma} \beta|^{2}}=1-\left|\frac{\gamma-\beta}{1-\bar{\gamma} \beta}\right|^{2} \leq 1, \quad \gamma, \beta \in \mathbb{D},
$$

we see that (5.6) is proved as soon as we show that

$$
\inf _{z \in \mathbb{D}} \frac{1-\left|b_{\lambda}(z)\right|^{2}}{|z-\lambda|^{2}}>0 .
$$

Note that condition (5.7) follows immediately from

$$
1-\left|b_{\lambda}(z)\right|^{2} \geq \frac{a_{0}|z-\lambda|^{2}}{\left|\lambda-a_{0} z\right|^{2}}, \quad z \in \mathbb{D} .
$$

To prove (5.8), we can of course assume that $\lambda=1$, and then it reduces to

$$
1-\left|b_{1}(z)\right|^{2} \geq \frac{a_{0}|z-1|^{2}}{\left|1-a_{0} z\right|^{2}}, \quad z \in \mathbb{D} .
$$

But using the identity $\left(1-a_{0}\right)^{2}=\alpha a_{0}$, we have

$$
\begin{aligned}
1-\left|b_{1}(z)\right|^{2} & =1-\frac{\alpha a_{0}|z|^{2}}{\left|1-a_{0} z\right|^{2}}=\frac{\left|1-a_{0} z\right|^{2}-\alpha a_{0}|z|^{2}}{\left|1-a_{0} z^{2}\right|} \\
& =\frac{1+a_{0}^{2}|z|^{2}-2 a_{0} \Re(z)-\alpha a_{0}|z|^{2}}{\left|1-a_{0} z\right|^{2}} .
\end{aligned}
$$

Therefore (5.9) is equivalent to

$$
1+a_{0}^{2}|z|^{2}-2 a_{0} \Re(z)-\alpha a_{0}|z|^{2} \geq a_{0}\left(1+|z|^{2}-2 \Re(z)\right)
$$

or

$$
1-a_{0}+a_{0}\left(a_{0}-\alpha-1\right)|z|^{2} \geq 0 .
$$

Using now the relation $a_{0}\left(a_{0}-\alpha-1\right)=a_{0}-1$, we obtain immediately the last inequality for every $z \in \mathbb{D}$. That proves (5.9) and the result.

Remark 5.2. In [4], a reproducing kernel thesis was proved for the embedding of $\mathcal{H}(b)$ into $L^{2}(\nu)$ in the case where the function $b$ satisfied some additional hypothesis. More precisely, assume that $b$ satisfies the so-called connected level set condition (which means that for some $\varepsilon \in(0,1)$, the level set $\Omega(b, \varepsilon):=\{z \in \mathbb{D}:|b(z)|<\varepsilon\}$ is connected) and assume further that the spectrum of $b, \sigma(b)=\left\{\zeta \in \mathbb{T}: \liminf _{z \rightarrow \zeta}|b(z)|<1\right\}$, is contained in the closure of $\Omega(b, \varepsilon)$. Then the operator $f \mapsto f$ is bounded from $\mathcal{H}(b)$ into $L^{2}(\nu)$ if and only if it is bounded on reproducing kernels.

In our case (which corresponds to a Dirichlet-type space), the hypothesis $\sigma\left(b_{\lambda}\right) \subset$ $\operatorname{Clos} \Omega\left(b_{\lambda}, \varepsilon\right)$ is not satisfied, whence we cannot apply [4, Theorem 6.8] to get Theorem 5.1. Indeed, we easily check that $\sigma\left(b_{\lambda}\right)=\mathbb{T} \backslash\{\lambda\}$; now if $\sigma\left(b_{\lambda}\right) \subset \operatorname{Clos} \Omega\left(b_{\lambda}, \varepsilon\right)$, then we would have $\lambda \in \operatorname{Clos} \Omega\left(b_{\lambda}, \varepsilon\right)$, but this is absurd by the continuity of $b_{\lambda}$ on the closed unit disk. 


\section{§6. REPROducing KERNEL THESIS FOR $\mu=\sum_{j=1}^{n} \alpha_{j} \delta_{\lambda_{j}}$}

As has already been mentioned, in the case where $\mu$ is a finite sum of point masses, an explicit formula for the reproducing kernels is not known (except for one point mass). The reproducing kernel thesis can however be shown without such an explicit formula by using some techniques from [8]. The main idea is to relate the reproducing kernels in this case to the reproducing kernels in the case in which $\mu$ is an atomic measure and then use Theorem 5.1 .

Theorem 6.1. Let $\nu$ be a finite positive Borel measure on $\mathbb{D}$, and let $\mu=\sum_{j=1}^{n} \alpha_{j} \delta_{\lambda_{j}}$, $\lambda_{j} \in \mathbb{T}, \alpha_{j}>0$. Then $\nu$ is a Carleson measure for $\mathcal{D}(\mu)$ if and only if there exists a constant $C>0$ such that

$$
\int_{\mathbb{D}}\left|k_{w}^{\mu}\right|^{2} d \nu \leq C\left\|k_{w}^{\mu}\right\|_{\mu}^{2}
$$

for every $w \in \mathbb{D}$, where $k_{w}^{\mu}$ is the reproducing kernel for $\mathcal{D}(\mu)$ at $w$.

Before proving this theorem, we need some preliminary results. First, we will need a general result about complete Nevannlina-Pick reproducing kernels. Recall that a reproducing kernel $k$ on the unit disk is a complete Nevannlina-Pick kernel (complete $N P$ kernel) if $k_{0}(z)=1$ for all $z \in \mathbb{D}$ and there exists a sequence of analytic functions $\left\{b_{n}\right\}_{n \geq 1}$ on $\mathbb{D}$ such that

$$
1-\frac{1}{k_{\lambda}(z)}=\sum_{n \geq 1} b_{n}(z) \overline{b_{n}(\lambda)} \quad \text { for all } \lambda, z \in \mathbb{D} .
$$

This condition is equivalent to the assumption that $1-1 / k$ is positive definite. Shimorin in 29] showed that the $\mathcal{D}(\mu)$-spaces have a complete NP kernel. The first result we will need is due to McCullough and Trent [17. We will say that a subspace $\mathcal{M}$ of a Hilbert space $H$ is a multiplier invariant subspace if $\varphi \mathcal{M} \subset \mathcal{M}$ for every $\varphi \in M(H)$, the space of multipliers of $H$.

Theorem A (McCullough and Trent [17]). Let $k$ be a complete NP kernel and let $\mathcal{M}$ be a multiplier invariant subspace. Then there exists a sequence of multipliers $\left\{\varphi_{n}\right\} \subset \mathcal{M}$ such that

$$
P_{\mathcal{M}}=\sum_{n \geq 1} M_{\varphi_{n}} M_{\varphi_{n}}^{*}(S O T)
$$

where $P_{\mathcal{M}}$ denotes the projection onto $\mathcal{M}$, and $M_{\varphi_{n}}$ denotes the multiplication operator, $f \mapsto \varphi_{n} f$, and the series converges in the strong operator topology.

In particular, notice that if we take the function $k_{z}, z \in \mathbb{D}$, we have

$$
P_{\mathcal{M}} k_{z}=\sum_{n \geq 1} M_{\varphi_{n}} M_{\varphi_{n}}^{*} k_{z}
$$

Since $M_{\varphi_{n}}^{*} k_{z}=\overline{\varphi_{n}(z)} k_{z}$, for every $w \in \mathbb{D}$ we have

$$
P_{\mathcal{M}} k_{z}(w)=\sum_{n \geq 1} \varphi_{n}(w) \overline{\varphi_{n}(z)} k_{z}(w)
$$

or equivalently,

$$
\frac{P_{\mathcal{M}} k_{z}(w)}{k_{z}(w)}=\sum_{n \geq 1} \varphi_{n}(w) \overline{\varphi_{n}(z)}
$$

i.e., $\frac{P_{\mathcal{M}} k_{z}(w)}{k_{z}(w)}$ is positive definite.

We will also need the following result which is due to Richter and Sundberg [22, 20] and Aleman [2]. 
Theorem B. Let $\mathcal{M}$ be a multiplier invariant subspace of $\mathcal{D}(\mu)$. Then $\operatorname{dim}(\mathcal{M} \ominus z \mathcal{M})=$ 1 , and if $f \in \mathcal{M} \ominus z \mathcal{M}$ with $\|f\|_{\mu}=1$, then

(i) $|f(z)| \leq 1$ for all $z \in \mathbb{D}$;

(ii) $\|f g\|_{\mu}=\|g\|_{\mu_{f}}$, for every $g \in \mathcal{D}\left(\mu_{f}\right)$, where $d \mu_{f}=|f|^{2} d \mu$;

(iii) for every $g \in \mathcal{M}$, there exists $h \in \mathcal{D}\left(\mu_{f}\right)$ such that $g=f h$.

The following lemma, which already appeared in [8], is the key point in the proof of our theorem.

Lemma 6.2. Let $\mu=\sum_{j=1}^{n} \alpha_{j} \delta_{\lambda_{j}}, \lambda_{j} \in \mathbb{T}$ and $\alpha_{j}>0$. Then, for every $j=1, \ldots, n$, there exists a positive constant $a_{j}$ such that if $\mu_{j}=a_{j} \delta_{\lambda_{j}}$, then $\frac{k^{\mu_{j}}}{k^{\mu}}$ is positive definite.

Proof. First, notice that the kernel $k^{\mu}$ is never zero (see [28]) and consequently the quotient is well defined. Let $j \in\{1, \ldots, n\}$ be fixed and define

$$
\mathcal{M}_{j}:=\left\{f \in \mathcal{D}(\mu): f\left(\lambda_{i}\right)=0, \forall i \neq j\right\} .
$$

Then $\mathcal{M}_{j}$ is a multiplier invariant subspace of $\mathcal{D}(\mu)$. Take $\phi_{j} \in \mathcal{M}_{j} \ominus z \mathcal{M}_{j},\left\|\phi_{j}\right\|_{\mu}=1$. Then by Theorem B we see that the multiplication operator $M_{\phi_{j}}: \mathcal{D}\left(\mu_{\phi_{j}}\right) \rightarrow \mathcal{M}_{j}$ is an onto isometry (and consequently a unitary operator). Here,

$$
d \mu_{\phi_{j}}=\left|\phi_{j}\right|^{2} d \mu=\sum_{i=1}^{n} \alpha_{i}\left|\phi_{j}\left(\lambda_{i}\right)\right|^{2} \delta_{\lambda_{i}}=\alpha_{j}\left|\phi_{j}\left(\lambda_{j}\right)\right|^{2} \delta_{\lambda_{j}},
$$

the last equality following from the fact that $\phi_{j} \in \mathcal{M}_{j}$. Define $a_{j}:=\alpha_{j}\left|\phi_{j}\left(\lambda_{j}\right)\right|^{2}$ and $d \mu_{j}=d \mu_{\phi_{j}}$. Then the reproducing kernel for the space $\mathcal{M}_{j}$ is given by

$$
k_{z}^{\mathcal{M}_{j}}(w)=\overline{\phi_{j}(z)} \phi_{j}(w) k_{z}^{\mu_{j}}(w) .
$$

On the other hand, we also know that $k_{z}^{\mathcal{M}_{j}}=P_{\mathcal{M}_{j}} k_{z}^{\mu}$, hence

$$
\frac{k_{z}^{\mu_{j}}(w)}{k_{z}^{\mu}(w)}=\frac{1}{\overline{\phi_{j}(z)} \phi_{j}(w)} \frac{k_{z}^{\mathcal{M}_{j}}(w)}{k_{z}^{\mu}(w)}=\frac{1}{\overline{\phi_{j}(z)} \phi_{j}(w)} \frac{P_{\mathcal{M}_{j}} k_{z}^{\mu}(w)}{k_{z}^{\mu}(w)},
$$

and since each one of the factors is positive definite, the result follows.

Now we pass to the proof of the reproducing kernel thesis for the identity map $I$ : $\mathcal{D}(\mu) \rightarrow L^{2}(\nu)$, in the case where $\mu$ is a finite sum of point masses.

Proof of Theorem 6.1. Using Theorem 4.1 and Carleson's reproducing kernel thesis for $H^{2}$, it suffices to prove that if there exists a constant $C>0$ with

$$
\int_{\mathbb{D}}\left|k_{w}^{\mu}\right|^{2} d \nu \leq C\left\|k_{w}^{\mu}\right\|_{\mu}^{2}
$$

then

$$
\int_{\mathbb{D}} \frac{1}{|1-\bar{w} z|^{2}} \prod_{i=1}^{n}\left|z-\lambda_{i}\right|^{2} d \nu(z) \lesssim \frac{1}{1-|w|^{2}}
$$

Of course, it suffices to show that

$$
\frac{1-|w|^{2}}{|1-\bar{w} z|^{2}} \prod_{i=1}^{n}\left|z-\lambda_{i}\right|^{2} \lesssim \frac{\left|k_{w}^{\mu}(z)\right|^{2}}{\left\|k_{w}^{\mu}\right\|_{\mu}^{2}} .
$$

By Lemma 6.2, for every $j=1, \ldots, n$, there exists a positive constant $a_{j}$ such that if $\mu_{j}=a_{j} \delta_{\lambda_{j}}$, then $\frac{k^{\mu_{j}}}{k^{\mu}}$ is a positive definite function. In particular, that implies the 
inequality

$$
\left|\frac{k_{z}^{\mu_{j}}(w)}{k_{z}^{\mu}(w)}\right|^{2} \leq \frac{\left\|k_{z}^{\mu_{j}}\right\|_{\mu_{j}}^{2}\left\|k_{w}^{\mu_{j}}\right\|_{\mu_{j}}^{2}}{\left\|k_{z}^{\mu}\right\|_{\mu}^{2}\left\|k_{w}^{\mu}\right\|_{\mu}^{2}} .
$$

Now recall that by (5.5), there exists a constant $C>0$ such that for every $j$ we have

$$
\frac{1-|w|^{2}}{|1-\bar{w} z|^{2}}\left|z-\lambda_{j}\right|^{2} \leq C \frac{\left|k_{w}^{\mu_{j}}(z)\right|^{2}}{\left\|k_{w}^{\mu_{j}}\right\|_{\mu_{j}}^{2}} .
$$

Therefore, to show (6.2), it suffices to establish the following inequality for every $j$ :

$$
\prod_{i \neq j}\left|z-\lambda_{i}\right|^{2} \lesssim \frac{\left\|k_{z}^{\mu}\right\|_{\mu}^{2}}{\left\|k_{z}^{\mu_{j}}\right\|_{\mu_{j}}^{2}} .
$$

This is equivalent to the following inequality (obtained by using the notation of the proof of Lemma 6.2):

$$
\left\|P_{\mathcal{M}_{j}} k_{z}^{\mu}\right\|_{\mu}^{2} \prod_{i \neq j}\left|z-\lambda_{i}\right|^{2} \lesssim\left|\phi_{j}(z)\right|^{2}\left\|k_{z}^{\mu}\right\|_{\mu}^{2}
$$

On the other hand, notice that since the multiplication operator $M_{\phi_{j}}$ is unitary, the operator $M_{\phi_{j}^{-1}}: \mathcal{M}_{j} \rightarrow \mathcal{D}\left(\mu_{j}\right)$ is also unitary and satisfies $M_{\phi_{j}}^{*}=M_{\phi_{j}^{-1}}$. Therefore, if $f \in \mathcal{M}_{j}$, then $M_{\phi_{j}^{-1}} f \in \mathcal{D}\left(\mu_{j}\right) \subset H^{2}$.

Now, note that since $p_{j}(z):=\prod_{i \neq j}\left(z-\lambda_{i}\right)$ is a polynomial, we have $p_{j} \in H^{\infty} \cap \mathcal{D}\left(\mu_{\phi_{j}}\right)$, which means that $p_{j}$ is a multiplier of the space $\mathcal{D}\left(\mu_{j}\right)$ and consequently $p_{j} M_{\phi_{j}^{-1}} f \in$ $\mathcal{D}\left(\mu_{j}\right)$; this implies that $p_{j} M_{\phi_{j}^{-1}} f \in \mathcal{D}\left(\delta_{\lambda_{j}}\right)$.

If $i \neq j$, then, since $f=\phi_{j} \widetilde{f}$ for some $\tilde{f} \in \mathcal{D}\left(\mu_{j}\right) \subset H^{2}$, by (2.2) we have

$$
\begin{aligned}
\mathcal{D}_{\lambda_{i}}\left(p_{j} \phi_{j}^{-1} f\right) & =\frac{1}{2 \pi} \int_{0}^{2 \pi}\left|\frac{1}{e^{i t}-\lambda_{i}}\left(\frac{p_{j}\left(e^{i t}\right) f\left(e^{i t}\right)}{\phi_{j}\left(e^{i t}\right)}-\frac{p_{j}\left(\lambda_{i}\right) f\left(\lambda_{i}\right)}{\phi_{j}\left(\lambda_{i}\right)}\right)\right|^{2} d t \\
& =\frac{1}{2 \pi} \int_{0}^{2 \pi}\left|\prod_{\ell \neq i, j}\left(e^{i t}-\lambda_{\ell}\right) \tilde{f}\left(e^{i t}\right)\right|^{2} d t \lesssim\|\tilde{f}\|_{H^{2}}^{2}<+\infty,
\end{aligned}
$$

and consequently $p_{j} \phi_{j}^{-1} f \in \mathcal{D}\left(\delta_{\lambda_{i}}\right)$. Thus, $p_{j} \phi_{j}^{-1} f \in \bigcap_{i=1}^{n} \mathcal{D}\left(\delta_{\lambda_{i}}\right)=\mathcal{D}(\mu)$ and moreover, $p_{j} \phi_{j}^{-1} f \in \mathcal{M}_{j}$.

Therefore, the multiplication operator $M_{p_{j} \phi_{j}^{-1}}: \mathcal{M}_{j} \rightarrow \mathcal{M}_{j}$ is well defined and by the closed-graph theorem we conclude that it is bounded. Hence,

$$
\begin{aligned}
\frac{\left|p_{j}(z)\right|\left\|k_{z}^{\mathcal{M}_{j}}\right\|_{\mu}^{2}}{\left|\phi_{j}(z)\right|} & =\left|\left\langle M_{p_{j} \phi_{j}^{-1}}\left(k_{z}^{\mathcal{M}_{j}}\right), k_{z}^{\mu}\right\rangle_{\mu}\right| \\
& \leq\left\|M_{p_{j} \phi_{j}^{-1}} k_{z}^{\mathcal{M}_{j}}\right\|_{\mu}\left\|k_{z}^{\mu}\right\|_{\mu} \leq\left\|M_{p_{j} \phi_{j}^{-1}}\right\|\left\|k_{z}^{\mathcal{M}_{j}}\right\|_{\mu}\left\|k_{z}^{\mu}\right\|_{\mu},
\end{aligned}
$$

where $\left\|M_{p_{j} \phi_{j}^{-1}}\right\|$ denotes the norm of the operator $M_{p_{j} \phi_{j}^{-1}}$ acting on $\mathcal{M}_{j}$. This gives (6.5) and concludes the proof.

Remark 6.3. Let $0 \leq \alpha \leq 1$. The weighted Dirichlet space $\mathcal{D}_{\alpha}$ consists of analytic functions $f$ on $\mathbb{D}$ such that

$$
\|f\|_{\alpha}^{2}:=|f(0)|^{2}+\int_{\mathbb{D}}\left|f^{\prime}(z)\right|^{2}\left(1-|z|^{2}\right)^{\alpha} d A(z)<\infty .
$$


Note that $\mathcal{D}_{1}=H^{2}$ and $\mathcal{D}_{0}=\mathcal{D}$. The reproducing kernels in $\mathcal{D}_{\alpha}$ are given by

$$
k_{w}^{\alpha}(z)= \begin{cases}(1-\bar{w} z)^{-\alpha} & \text { if } 0<\alpha \leq 1 \\ \frac{1}{\bar{w} z} \log \left(\frac{1}{1-\bar{w} z}\right) & \text { if } \quad \alpha=0 .\end{cases}
$$

Suppose that a finite positive measure $\nu$ on $\mathbb{D}$ satisfies

$$
\int_{\mathbb{D}}\left|k_{w}^{\alpha}\right|^{2} d \nu \leq c\left\|k_{w}^{\alpha}\right\|_{\alpha}^{2}, \quad w \in \mathbb{D} .
$$

It is not difficult to see that (6.6) is equivalent to saying that $\nu$ is an $\alpha$-Carleson measure, i.e.,

$$
\nu(S(I))= \begin{cases}O\left(|I|^{\alpha}\right) & \text { if } 0<\alpha \leq 1 ; \\ O\left(\left(\log \frac{e}{|I|}\right)^{-1}\right) & \text { if } \alpha=0\end{cases}
$$

for every arc $I \subset \mathbb{T}$ (see for instance [3] or [27]). On the other hand, as noted in [16] (see also [27]), not all $\alpha$-Carleson measures are Carleson measures for $\mathcal{D}_{\alpha}$, for $\alpha \in[0,1)$. In other words, the (RKT) does fails in $\mathcal{D}_{\alpha}$ spaces, $\alpha \in[0,1)$.

\section{$\S 7$. REPRODUCING KERNEL THESIS AND COMPACTNESS}

One can ask whether the property of being a compact Carleson measure (the embedding $\mathcal{D}(\mu) \hookrightarrow L^{2}(\nu)$ is compact) could be also characterized by a property on the family of normalized reproducing kernels. Note that we should normalize the reproducing kernels to obtain a weakly null sequence. However, this is not sufficient as shown in the following result.

Lemma 7.1. Let $\mu=\sum_{j=1}^{n} \alpha_{j} \delta_{\lambda_{j}}, \lambda_{j} \in \mathbb{T}, \alpha_{j}>0$, let $\hat{k}_{w}^{\mu}=k_{w}^{\mu} /\left\|k_{w}^{\mu}\right\|_{\mu}$ be the normalized reproducing kernel for $\mathcal{D}(\mu)$ at $w$, and let $\zeta \in \mathbb{T}$. Then the $\widehat{k}_{w}^{\mu}$ converge weakly to zero as $w \rightarrow \zeta$ if and only if $\zeta \neq \lambda_{j}$ for $1 \leq j \leq n$.

Proof. We easily see that the normalized reproducing kernel $\widehat{k}_{w}^{\mu}$ converges weakly to zero as $w \rightarrow \zeta$ if and only if $\widehat{k}_{w}^{\mu}$ converges uniformly to zero on the compact subsets of $\mathbb{D}$ as $w \rightarrow \zeta$.

First, consider the case of one point mass, $\mu=\alpha \delta_{\lambda}, \lambda \in \mathbb{T}$ and $\alpha>0$. Note that

$$
\frac{k_{w}^{\mu}(z)}{\left\|k_{w}^{\mu}\right\|_{\mu}}=\frac{1-\overline{b_{\lambda}(w)} b_{\lambda}(z)}{1-\bar{w} z} \cdot\left(\frac{1-|w|^{2}}{1-\left|b_{\lambda}(w)\right|^{2}}\right)^{1 / 2},
$$

where $b_{\lambda}$ is the function in the unit ball of $H^{\infty}$ given by equation (5.1). Moreover, if $K$ is a compact subset of $\mathbb{D}$ and $z \in K$, we have

$$
\left|\frac{1-\overline{b_{\lambda}(w)} b_{\lambda}(z)}{1-\bar{w} z}\right| \asymp 1, \quad w \in \mathbb{D} .
$$

Then the $\widehat{k}_{w}^{\mu}$ converge weakly to zero as $w \rightarrow \zeta$ if and only if

$$
\lim _{w \rightarrow \zeta} \frac{1-|w|^{2}}{1-\left|b_{\lambda}(w)\right|^{2}}=0,
$$

or equivalently, $b_{\lambda}$ has no angular derivative at $\zeta$ (recall that $b_{\lambda}$ is said to have an angular derivative at point $\zeta$ if $b_{\lambda}$ and $b_{\lambda}^{\prime}$ have angular limits at $\zeta$ and $\left.\left|b_{\lambda}(\zeta)\right|=1\right)$. This is true if and only if $\zeta \in \mathbb{T} \backslash\{\lambda\}$.

For the general case, fix $j \in\{1, \ldots, n\}$ and note that (6.3) can be written as

$$
\left|\widehat{k}_{w}^{\mu_{j}}(z)\right| \leq\left|\widehat{k}_{w}^{\mu}(z)\right| \frac{\left\|k_{z}^{\mu_{j}}\right\|_{\mu_{j}}}{\left\|k_{z}^{\mu}\right\|_{\mu}}, \quad z, w \in \mathbb{D} .
$$


Now take $K$ to be a compact subset of $\mathbb{D}$. By (6.4), there exists a constant $C(K)>0$ such that for any $z \in K$, we have

$$
\frac{\left\|k_{z}^{\mu_{j}}\right\|_{\mu_{j}}}{\left\|k_{z}^{\mu}\right\|_{\mu}} \lesssim \frac{1}{\prod_{i \neq j}\left|z-\lambda_{i}\right|} \lesssim C(K) .
$$

Hence

$$
\left|\widehat{k}_{w}^{\mu_{j}}(z)\right| \lesssim C(K)\left|\widehat{k}_{w}^{\mu}(z)\right|, \quad z \in K .
$$

Now if $\hat{k}_{w}^{\mu}$ converges uniformly to zero on $K$ as $w \rightarrow \zeta$, the last inequality implies that $\hat{k}_{w}^{\mu_{j}}$ also converges uniformly to zero on $K$ as $w \rightarrow \zeta$. The first part of the proof now gives $\zeta \neq \lambda_{j}$.

Conversely, let $\zeta \neq \lambda_{j}, j=1, \ldots, n$, and let $f \in \mathcal{D}(\mu)$. We have

$$
\left\langle f, \widehat{k}_{w}^{\mu}\right\rangle_{\mu}=\frac{1}{\left\|k_{w}^{\mu}\right\|_{\mu}} f(w)=\frac{\left\|k_{w}^{\mu_{j}}\right\|_{\mu_{j}}}{\left\|k_{w}^{\mu}\right\|_{\mu}}\left\langle f, \widehat{k}_{w}^{\mu_{j}}\right\rangle_{\mu_{j}} .
$$

Using (6.4) once again, we get

$$
\left|\left\langle f, \widehat{k}_{w}^{\mu}\right\rangle_{\mu}\right| \lesssim \frac{1}{\prod_{i \neq j}\left|w-\lambda_{i}\right|}\left|\left\langle f, \widehat{k}_{w}^{\mu_{j}}\right\rangle_{\mu_{j}}\right| .
$$

But since $\zeta \neq \lambda_{j}$ for any $j=1, \ldots, n$, the first part of the proof shows that $\left\langle f, \widehat{k}_{w}^{\mu_{j}}\right\rangle_{\mu_{j}} \rightarrow 0$ and $\prod_{i \neq j}\left|w-\lambda_{i}\right|$ remains bounded below as $w \rightarrow \zeta$. Thus, $\left\langle f, \widehat{k}_{w}^{\mu}\right\rangle_{\mu} \rightarrow 0$ as $w \rightarrow \zeta$.

The following result shows now that the reproducing kernel thesis is true for the compactness of the embedding $\mathcal{D}(\mu) \hookrightarrow L^{2}(\nu)$ when $\mu$ is a finite sum of point masses.

Theorem 7.2. Let $\nu$ be a finite positive Borel measure on $\mathbb{D}$, and let $\mu=\sum_{j=1}^{n} \alpha_{j} \delta_{\lambda_{j}}$, where $\lambda_{j} \in \mathbb{T}$ and $\alpha_{j}>0$. Then $\nu$ is a compact Carleson measure for $\mathcal{D}(\mu)$ if and only if for any $\zeta \in \mathbb{T} \backslash\left\{\lambda_{1}, \ldots, \lambda_{n}\right\}$, we have

$$
\int_{\mathbb{D}}\left|k_{w}^{\mu}\right|^{2} d \nu=o\left(\left\|k_{w}^{\mu}\right\|_{\mu}^{2}\right), \quad w \rightarrow \zeta .
$$

Proof. By Lemma 7.1, condition (7.1) is necessary for $\nu$ to be a compact Carleson measure for $\mathcal{D}(\mu)$. Conversely, assume that (7.1) is satisfied for any $\zeta \neq \lambda_{j}, j=1, \ldots, n$. Using Theorem 4.3, we shall show that $d \sigma(z):=\prod_{i=1}^{n}\left|z-\lambda_{i}\right|^{2} d \nu(z)$ is a compact Carleson measure for $H^{2}$, which is equivalent (by Carleson's result) to

$$
\sigma(S(\zeta, h))=o(h), \quad h \rightarrow 0
$$

for any $\zeta \in \mathbb{T}$. First, we consider $\zeta \in \mathbb{T} \backslash\left\{\lambda_{1}, \ldots, \lambda_{n}\right\}$ and fix $j \in\{1, \ldots, n\}$. Using (6.3) and (6.4), we have

$$
\begin{aligned}
\frac{1}{\left\|k_{w}^{\mu}\right\|_{\mu}^{2}} \int_{\mathbb{D}}\left|k_{w}^{\mu}(z)\right|^{2} d \nu(z) & \geq \int_{\mathbb{D}} \frac{\left|k_{w}^{\mu_{j}}(z)\right|^{2}\left\|k_{z}^{\mu}\right\|_{\mu}^{2}}{\left\|k_{w}^{\mu_{j}}\right\|_{\mu_{j}}^{2}\left\|k_{z}^{\mu_{j}}\right\|_{\mu_{j}}^{2}} d \nu(z) \\
& \gtrsim \frac{1}{\left\|k_{w}^{\mu_{j}}\right\|_{\mu_{j}}^{2}} \int_{\mathbb{D}}\left|k_{w}^{\mu_{j}}(z)\right|^{2} \prod_{i \neq j}\left|z-\lambda_{j}\right|^{2} d \nu(z) .
\end{aligned}
$$

But we know that

$$
\frac{\left|k_{w}^{\mu_{j}}(z)\right|^{2}}{\left\|k_{w}^{\mu_{j}}\right\|_{\mu_{j}}^{2}}=\frac{1-|w|^{2}}{1-\left|b_{\lambda_{j}}(w)\right|^{2}} \frac{\left|1-\overline{b_{\lambda_{j}}(w)} b_{\lambda_{j}}(z)\right|^{2}}{|1-\bar{w} z|^{2}},
$$

and by (5.6), there exists a constant $\delta>0$ such that for any $z, w \in \mathbb{D}$ we have

$$
\frac{\left|1-\overline{b_{\lambda_{j}}(w)} b_{\lambda_{j}}(z)\right|^{2}}{1-\left|b_{\lambda_{j}}(w)\right|^{2}} \geq \delta\left|z-\lambda_{j}\right|^{2}
$$


Thus, we get

$$
\frac{1}{\left\|k_{w}^{\mu}\right\|_{\mu}^{2}} \int_{\mathbb{D}}\left|k_{w}^{\mu}(z)\right|^{2} d \nu(z) \gtrsim\left(1-|w|^{2}\right) \int_{\mathbb{D}} \frac{1}{|1-\bar{w} z|^{2}} d \sigma(z)
$$

and condition (7.1) gives

$$
\lim _{w \rightarrow \zeta}\left(\left(1-|w|^{2}\right) \int_{\mathbb{D}} \frac{1}{|1-\bar{w} z|^{2}} d \sigma(z)\right)=0
$$

for any $\zeta \in \mathbb{T} \backslash\left\{\lambda_{1}, \ldots, \lambda_{n}\right\}$. Now, a standard argument shows that (7.2) is satisfied for $\zeta \neq \lambda_{i}, i=1, \ldots, n$. Indeed, take $w=(1-h) \zeta$. Then, on one hand, for $z \in S(\zeta, h)$ we have

$$
|1-\bar{w} z|=|1-(1-h) \bar{\zeta} z|=|\zeta-(1-h) z| \leq|\zeta-z|+h \leq 2 h .
$$

On the other hand, $1-|w|^{2} \geq 1-|w|=h$. Then

$$
\frac{1}{4 h} \sigma(S(\zeta, h)) \leq\left(1-|w|^{2}\right) \int_{\mathbb{D}} \frac{1}{|1-\bar{w} z|^{2}} d \sigma(z) \rightarrow 0 \quad \text { as } h \rightarrow 0,
$$

which gives (7.2). It remains to observe that condition (7.2) for $\zeta=\lambda_{i}, i=1, \ldots, n$, is a consequence of the following trivial estimate:

$$
\sigma\left(S\left(\lambda_{i}, h\right)\right)=\int_{S\left(\lambda_{i}, h\right)} \prod_{j=1}^{n}\left|z-\lambda_{j}\right|^{2} d \nu(z) \leq 4^{n-1} h^{2}\|\nu\| .
$$

\section{REFERENCES}

[1] J. Agler, A disconjugacy theorem for Toeplitz operators, Amer. J. Math. 112 (1990), no. 1, 1-14. MR 1037599 (91b:47048)

[2] A. Aleman, The multiplication operators on Hilbert spaces of analytic functions, Habilitationsschrift, Fernuniv. Hagen, 1993.

[3] N. Arcozzi, D. Blasi, and J. Pau, Interpolating sequences on analytic Besov type spaces, Indiana Univ. Math. J. 58 (2009), no. 3, 1281-1318. MR2541368 (2010e:30044)

[4] A. Baranov, E. Fricain, and J. Mashreghi, Weighted norm inequalities for de Branges-Rovnyak spaces and their applications, Amer. J. Math. 132 (2010), no. 1, 125-155. MR2597508(2011a:46047)

[5] O. Blasco, Operators on weighted Bergman spaces $(0<p \leq 1)$ and applications, Duke Math. J. 66 (1992), no. 3, 443-467. MR.1167102 (93h:47036)

[6] L. Carleson, Interpolations by bounded analytic functions and the corona problem, Ann. of Math. (2) 76 (1962), 547-559. MR0141789 (25:5186)

[7] G. R. Chacón, Carleson measures on Dirichlet-type spaces, Proc. Amer. Math. Soc. 139 (2011), no. 5, 1605-1615. MR2763750 (2012h:42032)

[8] _ Interpolating sequences in harmonically weighted Dirichlet spaces, Integral Equations Operator Theory 69 (2011), 73-85. MR2749449 (2011m:31012)

[9] R. Chartrand, Toeplitz operators on Dirichlet-type spaces, J. Operator Theory 48 (2002), 3-13. MR.1926041 (2003g:47046)

[10] Multipliers and Carleson measures for $\mathfrak{D}(\mu)$, Integral Equations Operator Theory 45 (2003), 309-318. MR1965337 (2004b:30088)

[11] N. Chevrot, D. Guillot, and T. Ransford, De Branges-Rovnyak spaces and Dirichlet spaces, J. Funct. Anal. 259 (2010), no. 9, 2366-2383. MR2674117 (2012m:46029)

[12] C. Fefferman and E. M. Stein, $H^{p}$ spaces of several variables, Acta Math. 129 (1972), no. 3-4, 137-193. MR0447953(56:6263)

[13] J. Garnett, Bounded analytic functions, Grad. Texts in Math., vol. 236, Springer, New York, 2007. MR.2261424(2007e:30049)

[14] V. P. Havin and N. K. Nikolski, Stanislav Aleksandrovich Vinogradov, his life and mathematics, Complex Analysis, Operators, and Related Topics, Oper. Theory Adv. Appl., vol. 113, Birkhäuser, Basel, 2000, pp. 1-18. MR1771747 (2001b:01025a)

[15] P. W. Jones, Carleson measures and the Fefferman-Stein decomposition of BMO(R), Ann. of Math. (2) 111 (1980), no. 1, 197-208. MR558401 (81d:30059)

[16] D. E. Marshall and C. Sundberg, Interpolating sequences for the multipliers of the Dirichlet space, Manuscript, 1994; Avalaible at http://www.math.washington.edu/marshall/preprints/ preprints.html. 
[17] S. McCullough and T. Trent, Invariant subspaces and Nevanlinna-Pick kernels, J. Funct. Anal. 178 (2000), 226-249. MR 1800795 (2002b:47006)

[18] N. K. Nikolski, Operators, functions, and systems: an easy reading. Vol. 2, Math. Surveys Monogr., vol. 93, Amer. Math. Soc., Providence, RI, 2002. MR 1892647 (2003i:47001b)

[19] S. Richter, Invariant subspaces of the Dirichlet shift, J. Reine Angew. Math. 386 (1988), 205-220. MR.936999 (89e:47048)

[20] S. Richter and C. Sundberg, Multipliers and invariant subspaces in the Dirichlet space, J. Operator Theory 28 (1992), 167-186. MR1259923 (95e:47007)

[21] S. Richter, A representation theorem for cyclic analytic two-isometries, Trans. Amer. Math. Soc. 328 (1991), no. 1, 325-349. MR1013337 (92e:47052)

[22] S. Richter and C. Sundberg, A formula for the local Dirichlet integral, Michigan Math. J. 38 (1991), no. 3, 355-379. MR 1116495 (92i:47035)

[23] D. Sarason, Sub-Hardy Hilbert spaces in the unit disc, Univ. Arkansas Lecture Notes in Math. Sci., vol. 10, Wiley, New York, 1994. MR.1289670 (96k:46039)

[24] L L L Lal Dirichlet spaces as de Branges-Rovnyak spaces, Proc. Amer. Math. Soc. 125 (1997), no. 7, 2133-2139. MR1396993 (98h:46023)

[25] _ Harmonically weighted Dirichlet spaces associated with finitely atomic measures, lntegral Equations Operator Theory 31 (1998), 186-213. MR.1623461 (99i:46015)

[26] A. Serra, Interpolating sequences in harmonically weighted Dirichlet spaces, Proc. Amer. Math. Soc. 131 (2003), 2809-2817. MR.1974338(2004b:30069)

[27] K. Seip, Interpolation and sampling in spaces of analytic functions, Univ. Lecture Ser., vol. 33, Amer. Math. Soc., Providence, RI, 2004. MR2040080 (2005c:30038)

[28] S. Shimorin, Reproducing kernels and extremal functions in Dirichlet-type spaces, Zap. Nauchn. Sem. S.-Peterburg. Otdel. Mat. Inst. Steklov. (POMI) 255 (1998), 198-220; English transl., J. Math. Sci. (N. Y.) 107 (2001), no. 4, 4108-4124. MR.1692900 (2000g:46034)

[29] _ Complete Nevanlinna-Pick property of Dirichlet-type spaces, J. Funct. Anal. 191 (2002), 276-296. MR 1911187 (2003c:47032)

[30] D. Stegenga, Multipliers of the Dirichlet space, Illinois J. Math. 24 (1980), no. 1, 113-139. $\operatorname{MR} 550655(81 \mathrm{a}: 30027)$

Departamento de Matematicas, Pontificia Universidad Javeriana, Cra. 7 No. 43-82, Bogotá, COLOMBIA

E-mail address: chacong@javeriana.edu.co

Laboratoire Paul Painlevé, UMR 8524, Université Lille 1, 59655 Villeneuve d’Ascq Cedex, FRANCE

E-mail address: emmanuel.fricain@math.univ-lille1.fr

Department of Engineering Science, College of Engineering, University of Tehran, TehRAN 11155-4563, IRAN

E-mail address: mahmood.shabankhan@gmail.com

Received 16/FEB/2012

Originally published in English 